\title{
Estimation of Oxygen Diffusing Conductance in Different Dissection Portion of the Placental Cotyledon of Passive Smoking Mothers \\ KN Ramesh ${ }^{1}$, AA Nasaruddin ${ }^{2}$, J Stanslas ${ }^{3}$, N Sivalingam ${ }^{4}$, I Mathavan ${ }^{5}$
}

\begin{abstract}
A study was undertaken to estimate the amount oxygen that can diffuse across the placenta of the passive smoking mothers in an Indo-Asian population. A total of 24 human term placentas, 11 from mothers exposed to tobacco smoke and 13 from those not exposed to tobacco smoke were collected for estimating weight, surface area and volume term placentas. Five placentas from each of the groups were used for estimating the various parameters for calculating the oxygen-diffusing conductance. A random sampling technique at 7 levels of tissue preparation was adopted. Cotyledons were cut into blocks and each block was further cut into upper (maternal), middle (foeto-maternal) and lower (foetal) components. Semithin sections, $1 \mu \mathrm{m}$ thick, stained using a modified toluidine was viewed and the images were analysed. A Merz grid was used to estimate the vascular volumes, exchange surface areas and tissue diffusion distances for calculation the oxygen-diffusing conductance of the placenta. These were combined with physicochemical quantities (oxygen-haemoglobin reaction rates and tissues oxygen diffusion coefficients) obtained from literature in order to estimate the partial conductance of the six tissue compartments of the oxygen pathway. The present study demonstrates that there is no significant difference in the total oxygen-diffusing conductance in the placenta of passive smokers and those not exposed to tobacco smoke. However, the significant increase in the foetal capillaries and foetal erythrocytes conductance suggests that an adaptive mechanism in the foetal part of the placenta may be occurring to ensure adequate oxygen to the foetus
\end{abstract}

Keywords: Indo-Asian population, oxygen-diffusing conductance, passive smoking mothers, placental cotyledons

From: ${ }^{1}$ Tuanku Ja'afar Hospital, Jalan Rasah, 70300 Seremban, Negeri Sembilan, Malaysia ${ }^{2}$ Faculty of Medicine Asia Metropolitan University G-8, Jalan Kemacahaya 11, Taman Kemacahaya 43200 Cheras Selangor, Malaysia, ${ }^{3}$ Pharmacotherapeutics Unit, Department of Medicine, Faculty of Medicine and Health Sciences, Universiti Putra Malaysia 43400 Serdang, Selangor, Malaysia ${ }^{4}$ Department of Obstetrics and Gynecology, Clinical School, International Medical University, Jalan Rasah, 70300 Seremban, Negeri Sembilan, Malaysia and ${ }^{5}$ Membrane Protein Lab, Diamond Light Source, Oxford, UK.

Correspondence: Dr R Nambiar, Tuanku Ja'afar Hospital, Jalan Rasah 70300 Seremban Negeri Sembilan, Malaysia, Fax: +606-767-9806, e-mail: rameshknambiar@yahoo.com 


\section{INTRODUCTION}

The placenta, a vital interface component between mother and fetus is important for nutrients and gas exchange, waste disposal, immunity and organ maturation (1). Placenta provides a large surface area for nutrients exchange to occur between the mother and fetus (2). Placental membranes are important for feto maternal nutrients exchange since the nutrients must pass a myriad of membranes (syncytiotrophoblast, cytotrophoblast, stroma, vascular endothelium), which present barriers between the mother and fetus blood circulation (3). The passage of nutrients across these barriers are dependent on physical and chemical properties (4).

There are many modes of nutrient and gas transport, which include diffusion, pinocytosis, and active or facilitated transport (5). The transport of oxygen is a diffusion-limited process since it is dependent on the oxygen pressure gradient between mother and fetus blood (6). Physiologists express the ability of an organ to act in oxygen diffusion exchange as oxygen conductance. This denotes the volume flux of oxygen per minute over a given partial pressure gradient (7). Transplacentaloxgen flow can be measured using a morphometric model formulated by Mayhew et al in 1984 (8). Thismodel measures the total resistance in six tissue compartments of placenta, which reflectsthe microstructure of the diffusion pathway $(8,9)$. The estimation of total resistance is the reciprocal to the total oxygen conductance (Dp) (9). This morphometric model has been successfully used in many placenta studies (7-11).

The adverse effect of passive smoking leading to pregnancy complication and mortality is well documented (12-14). Cigarette smoke contains more than 3000 toxic substances that result in damage of the placenta particularly in the membrane compartments (15). Strong evidence indicates that smoking cause profound changes to membrane thickness, vessel size and number of capillaries that is important in determining placental oxygen diffusive conductance $(9,16)$. However most 
studies have been conducted in the placenta of active or passive smoking mothers in developed countries $(12,13,17)$.

There is, however, a paucity of information on amount of oxygen can diffuse across the placenta of the passive smoking mothers in an Indo-Asian population. The present study was initiated to fill the gap that may exist in this area of research of the placenta. Furthermore, it will be interesting to measure the oxygen conductance in different dissection of placental cotyledon of passive smoking mothers as the cotyledons with its villous components are important for nutrients and oxygen transfers between the fetus bloods in the capillaries (6).

\section{SUBJECTS AND METHODS}

A total of 24 fresh placentas (10-15 minutes afterbirth) were collected from the Kuala Lumpur Maternity Hospital with approval from the hospital ethics committee in accordance with the Helsinki declaration. The study design was observational based and stratified sampling method was used to select the sample randomly for histological study. Informed consent was obtained from all the admitted mothers and a questionnaire was distributed before the study. The passive smoking is defined as mothers who are exposed to 5-14 cigarettes per day from their husband or close associates. The placenta collected comprised of 13 non-exposed and 11 exposed to tobacco smoke.

The rate of exposure was assessed subjectively (from volunteered cigarette exposure) and objectively (determining levels of urine cotinine, a major metabolite of nicotine). All the infants whose placenta was taken for the study were live-born, full term and no detectable congenital malformations. The exclusion criteria include pregnant women with no diabetes, hypertension and 
pregnancy complication that would lead to fetal and placental growth disorder. The collection and measurement of placental parameters were done according to established method. The umbilical cord was clamped at the placental insertion immediately after delivery and the cot was cut 5-20 $\mathrm{mm}$ from the insertion point before the membranes were trimmed away and the blood clots removed.

Urine cotinine level was determined using a HPLC method to estimate maternal smoking pattern(18). The urine sample was collected from the pregnant women immediately prior to delivery ( 0.25 -1hour before childbirth). The cotinine was extracted from urine using quinolone as internal standard and run in a HPLC system with a mobile phase flow rate of $1.5 \mathrm{ml} / \mathrm{min}$ and absorbance at $254 \mathrm{~nm}$. The method to measure oxygen diffusion was adapted from a wellestablished protocol $(8,9)$. The capacity for diffusion is expressed as a diffusive conductance, Dp, in $\mathrm{mlO}_{2} / \mathrm{min} / \mathrm{kPa}(9)$.

The cotyledons were portioned into upper, middle and lower section using a point grid system. Systematic random sampling procedures were adopted in all levels of samplings such as blocks, sections field of view and individual measurement. The conductance for each tissue layers was carried out by stereology method and physicochemical variables. For stereological studies, the light microscope and Merz grid were used. Results were assessed statistically by analyses of variance (ANOVA) and Student's t-test. The form of t-test used was an independent samples twotailed t test. Significance was set at the $\mathrm{p}<0.05$ levels Statistical analyses were executed by using SPSS for Windows (Version 9.0, SPSS Inc. Chicago, Illinois, USA). 


\section{RESULTS}

The mean age of all participating mothers were $28.6 \pm 4.76$ years and the gestational age between 37-42 weeks (Table 1). For the group of smoking exposed mothers, the mean height was $1.57 \mathrm{~m}$, the mean weight on admission was $62.6 \mathrm{~kg}$ and the mean body mass index (BMI) was $25.5 \mathrm{~kg} / \mathrm{m}^{2}$ respectively. No significant differences were observed in non-exposed groups I in comparison with the exposed groups for all the variables mentioned (Table 1).

Urine cotinine determination by HPLC method shows that the mean cotinine concentration was not significantly different between exposed $(17.10 \pm 15.65)$ and non-exposed $(10.11 \pm 7.50)$ groups (Table 1). The gross surface differences of normal and passive smokers placenta showed some interesting observations. The entire passive smokers placenta showed the presence of blood clot (abruption) on the cotyledons surface while three of the smokers showed calcification and multiple infarction on the surface. There is no difference in the placenta size between and passive and normal mothers (Table 1). The apparent increase in placental surface area, weight and specific weight in the exposed group was not statistically significant. However, we did observe a significantly higher placental volume $(\mathrm{p}<0.05)$ in the exposed group (mean 491.2, CI: 446.7535.6) compared to non-exposed group (mean 411.5, CI: 363.6-459.3) (Table 1).

The structural variables for oxygen conductance determination are shown in Table 2 . Maternal-intervillous spaces and foetal-intervillous spaces of the three region of cotyledon (upper, middle and lower) showed no significant difference between exposed and non-exposed groups. The exchange surface area of maternal erythrocytes in the upper region of the cotyledon was significantly higher $(\mathrm{p}<0.05)$ in the exposed group. However, exchange surface area in the middle and lower regions of the cotyledon were not significantly different. A significant difference $(\mathrm{p}<$ 0.05) was observed in the surface area of maternal aspect of the trophoblast in the lower region of 
the cotyledon for the exposed group. The exchange surface area of foetal aspect of trophoblast, luminal aspect of foetal capillaries and foetal erythrocytes showed no significant difference between exposed and non-exposed group in all the regions of the cotyledon. Moreover, we found no significant difference in the harmonic mean thickness of maternal plasma, trophoblast and stroma for all the regions of the cotyledon in both exposed and non-exposed groups. Interestingly, we found that the harmonic mean thickness of foetal plasma in the upper region of the cotyledon was significantly higher $(\mathrm{p}<0.05)$ in the exposed group.

Partial diffusing conductance of foetal plasma in all three regions of the cotyledon was not significantly different between exposed and non-exposed groups. However, when the foetal erythrocytes were taken into consideration, the partial diffusing conductance in the lower region of the cotyledon was significantly $(\mathrm{p}<0.05)$ higher in the exposed groups. The total diffusive conductance of the placentae in all the regions of the cotyledons was significantly different between exposed and non-exposed groups. Furthermore, the specific diffusive conductance of the placental oxygen pathway in all three regions was not significantly different. The mean conductance in both exposed and non-exposed group was in the range of $10-15 \mathrm{ml} / \mathrm{min} / \mathrm{kPa} / \mathrm{kg}$. In exposed groups, the regression equation for the mean total conductance against birth weight took the allometric form $\mathrm{Dp}=24.56 \mathrm{~W}^{5.77}$ with no significant difference.

The partial relative resistance of maternal erythrocytes, maternal plasma, trophoblast and stromal layers in all three regions of the cotyledon were not significantly different between exposed and non-exposed groups. The main contributors to total oxygen diffusive resistance were the trophoblast (46-48\%), maternal plasma (15-23\%) and stroma (16-19\%). In addition, foetal plasma accounted for 7-11\% and foetal erythrocytes about $4 \%$ of the total oxygen diffusive resistance. The number of villi was significantly higher $(\mathrm{p}<0.05)$ in the lower region of the 
cotyledon in the exposed group. In addition, the total number of villi was found to be higher in the exposed group compared to non-exposed group.

\section{DISCUSSION}

Gross pathological changes, such as placental abruption, infarction and calcifications have been observed on the placenta of those mothers who were exposed to tobacco smoke. This raises the possibility of foetal hypoxemia occurring in those women exposed to tobacco smoke during pregnancy (19). The non-significant alteration in the total oxygen conductance between exposed and non-exposed group is related to non-decrease birth weight in both group tested (9). The insignificant mean partial conductance of maternal erythrocytes and plasma could be attributed to the effects of carbon monoxide in the tobacco smoke. Carbon monoxide has a far higher affinity for hemoglobin than oxygen and thus reduces oxygen capacity of the maternal blood (functional anemia). Other studies have shown that the levels of carboxyhaemoglobin are greater in mothers exposed to tobacco smoke (20-22) and this factor is not accounted for in the morphometric model. Therefore, mean conductances of maternal erythrocytes and plasma, probably underestimated for exposed group.

Our findings further indicate that the upper portion of cotyledon comprised a proportionally lower volume of villous structure but a proportionally higher volume of maternal blood space, which are vice versa in lower portion of cotyledon. The present study also showed a significant increase in partial conductance of maternal plasma in upper region of cotyledon in the placenta of passive smokers. This suggests that there are greater surface areas of erythrocytes plasma membrane on this part of cotyledon. This finding varies with others, where it is in an 
agreement with (9) but in contrast with (23). Moreover, ours may be in advantage than the others since we have partitioned the cotyledons into three parts namely upper, middle and lower section. Our findings further indicate that the upper portion of cotyledon comprised a proportionally lower volume of villous structure but a proportionally higher volume of maternal blood space, which are vice versa in lower portion of cotyledon.

Trophoblast conductance was not altered in passive smoking group and this result could be contributed to the rate of exposure to tobacco smoke that is in the range of $15-30 \mathrm{ng} / \mathrm{ml}$. There is no significant increase in the harmonic mean distance across the villous stroma indicating that other factors might affect the conductance across the layers. The augmented value of foetal erythrocytes conductance and number of foetal capillaries in exposed group indicate that postplacental compensation may have taken place to improve diffusion in passive smokers placenta. The post-placental compensatory mechanism includes an increase in peripheral surface area due to the increase in number of foetal capillaries and profuse branching.

\section{CONCLUSION}

In conclusion this study has shown that passive smoking has an effect on placenta and significant diffusive conductance was found in different part within the cotyledon. Previous works have used the entire block of cotyledon to estimate oxygen-diffusing capacity in various condition of foetal hypoxic stress where as we divide the cotyledon into three parts namely maternal, materno-fetal and foetal part. Further investigation is needed to explore the compensatory mechanism of placenta in overcoming the hypoxic stress. The small number of the sample size warrants comment since we used convenient sampling method in a single hospital based environment. Most of our samples 
are low income group patients which do not represent the whole population. Factors such as short duration of project, funding and facilities are limiting factors to look into large number of placentae with ethnic stratification.

\section{ACKNOWLEDGMENT}

The authors thank the participants who willing to give their placentas to this project. The authors thank project staff (Siti Muskinah and Anatomy Unit) for their contributions to the project. Sincere gratitude to Prof MK Vidadaran Menon and Associate Prof Goh Yong Meng from Universiti Putra Malaysia. Special thank to Prof Malcolm Symonds and Prof TM Mayhew, University of Nottingham, Queen's Medical Centre, UK. The authors also thank Department of Medicine, Faculty of Medicine and Health Sciences, Universiti Putra Malaysia 43400 Serdang, Selangor, Malaysia and Department of Obstetrics \& Gynaecology, Hospital Kuala Lumpur for assistance with equipment for the project.

\section{AUTHOR'S NOTE}

Ramesh KN conceived paper, conducted research, oversaw data collection, wrote manuscript and approval final version. Nasaruddin AA participated in study design, data analysis and interpretation, revised manuscript and approval final version. Stanslas J participated in study design, interpretation of data and revision of manuscript and approval final version. Sivalingam $\mathrm{N}$ provided oversight to study, participated in data interpretation, and critically revised manuscript and approval final version. Mathavan I participated in study design, interpretation of data and 
Oxygen Diffusion Conductance in Placental Cotyledon of Passive Smoking Mothers

revision of manuscript and approval final version. The authors declare that they have no conflict of interest.

\section{REFERENCES}


1. Lobo SE, Leonel LC, Miranda CM, Coelho TM, Ferreira GA, Mess A et al. The Placenta as an Organ and a Source of Stem Cells and Extracellular Matrix: A Review. Cells Tissues Organs. 2016; 201: 239-52.

2. Burton GJ, Fowden AL. The placenta: a multifaceted, transient organ. Philos Trans R Soc Lond B Biol Sci. 2015; 370: 20140066.

3. Genbacev O, McMaster MT, Lazic J, Nedeljkovic S, Cvetkovic M, Joslin R et al. Concordant in situ and in vitro data show that maternal cigarette smoking negatively regulates placental cytotrophoblast passage through the cell cycle. Reprod Toxicol. 2000; 14: $495-506$.

4. Prouillac C, Lecoeur S. The role of the placenta in fetal exposure to xenobiotics: importance of membrane transporters and human models for transfer studies. Drug Metab Dispos. 2010; 38: 1623-35.

5. Brett KE, Ferraro ZM, Yockell-Lelievre J, Gruslin A, Adamo KB. Maternal-fetal nutrient transport in pregnancy pathologies: the role of the placenta. Int J Mol Sci 2014; 15: 1615385.

6. Guttmacher AE, Maddox YT, Spong CY. The Human Placenta Project: placental structure, development, and function in real time. Placenta 2014; 35: 303-4.

7. Mayhew TM. Estimating oxygen diffusive conductances of gas-exchange systems: A stereological approach illustrated with the human placenta. Ann Anat 2014; 196: 34-40.

8. Mayhew TM, Joy CF, Haas JD. Structure-function correlation in the human placenta: the morphometric diffusing capacity for oxygen at full term. J Anat. 1984; 139( Pt 4): 691708. 
9. Bush PG, Mayhew TM, Abramovich DR, Aggett PJ, Burke MD, Page KR. Maternal cigarette smoking and oxygen diffusion across the placenta. Placenta.2000; 21: 824-33.

10. Mayhew TM, Jackson MR, Haas JD. Oxygen diffusive conductances of human placentae from term pregnancies at low and high altitudes. Placenta 1990; 11: 493-503.

11. Mayhew TM, Jackson MR, Boyd PA. Changes in oxygen diffusive conductances of human placentae during gestation (10-41 weeks) are commensurate with the gain in fetal weight. Placenta. 1993; 14: 51-61.

12. Zhang L, Hsia J, Tu X, Xia Y, Zhang L, Bi Z et al. Exposure to secondhand tobacco smoke and interventions among pregnant women in China: a systematic review. Prev Chronic Dis 2015; 12: E35.

13. Ion R, Bernal AL. Smoking and Preterm Birth Reprod Sci 2015; 22: 918-26.

14. Hawsawi AM, Bryant LO, Goodfellow LT. Association between exposure to secondhand smoke during pregnancy and low birthweight: a narrative review. Respir Care 2015; 60: $135-40$.

15. Behera SN, Xian H, Balasubramanian R. Human health risk associated with exposure to toxic elements in mainstream and sidestream cigarette smoke. Sci Total Environ 2014; 472: $947-56$.

16. Mayhew TM, Sisley I. Quantitative studies on the villi, trophoblast and intervillous pores of placentae from women with well-controlled diabetes mellitus. Placenta 1998; 19 (5-6): $371-7$.

17. Urbaniak T, Klejewski A, Sobczyk K. [Influence of smoking on pregnency course and fetal development]. Przegl Lek 2015; 72: 144-7. 
18. Greaves R, Trotter L, Brennecke S, Janus E. A simple high-pressure liquid chromatography cotinine assay: validation of smoking status in pregnant women. Ann Clin Biochem. 2001; 38(Pt 4): 333-8.

19. Kaminsky LM, Ananth CV, Prasad V, Nath C, Vintzileos AM. The influence of maternal cigarette smoking on placental pathology in pregnancies complicated by abruption. Am J Obstet Gynecol 2007; 197: 275 e1-5.

20. Harrison KL, Robinson AG. The effect of maternal smoking on carboxyhemoglobin levels and acid-base balance of the fetus. Clin Toxicol 1981; 18: 165-8.

21. Manning FA, Feyerabend C. Cigarette smoking and fetal breathing movements. Br J Obstet Gynaecol 1976; 83: 262-70.

22. Bureau MA, Monette J, Shapcott D, Pare C, Mathieu JL, Lippe J et al. Carboxyhemoglobin concentration in fetal cord blood and in blood of mothers who smoked during labor. Pediatrics 1982; 69: 371-3.

23. Bush PG, Mayhew TM, Abramovich DR, Aggett PJ, Burke MD, Page KR. A quantitative study on the effects of maternal smoking on placental morphology and cadmium concentration Placenta 2000; 21: 247-56. 
Table 1: Maternal, neonatal and placental variables in exposed and non-exposed subjects

\begin{tabular}{llllc}
\hline Variable & Exposed & Non-exposed & df & p level \\
\hline Maternal & & & & \\
Height $(\mathrm{m})$ & $1.57(1.54-1.60)$ & $1.56(1.53-1.59)$ & 22 & 0.726 \\
Weight $(\mathrm{kg})$ & $62.6(57.7-67.5)$ & $65.0(58.1-72.0)$ & 22 & 0.565 \\
BMI $\left(\mathrm{kg} / \mathrm{m}^{2}\right)$ & $25.5(22.9-28.0)$ & $26.6(24.2-28.9)$ & 22 & 0.498 \\
Neonatal & & & & \\
Gestational age(days) & $276.4(270.0-282.8)$ & $278.2(273.4-282.9)$ & 22 & 0.621 \\
Birth weight $(\mathrm{kg})$ & $3.17(2.99-3.36)$ & $3.16(2.94-3.38)$ & 22 & 0.92 \\
Length $(\mathrm{m})$ & $0.47(0.46-0.50)$ & $0.48(0.46-0.50)$ & 22 & 0.648 \\
BMI kg/m & $14.1(13.1-15.1)$ & $13.7(12.8-14.6)$ & 22 & 0.501 \\
Head circumference $(\mathrm{cm})$ & $32.9(32.3-33.5)$ & $33.0(32.3-33.8)$ & 22 & 0.852 \\
& & & & \\
Urine cotinine(ng/ml) & $17.1 \pm 15.65$ & $10.11 \pm 7.50$ & 22 & 0.824 \\
Surface area m ${ }^{2}$ & $324.4(292.3-355.7)$ & $287.4(242.6-334.3)$ & 22 & 0.197 \\
Volume $\left(\mathrm{cm}^{3}\right)$ & $491.2(446.7-535.6)$ & $411.5(363.6-459.3)$ & 22 & $0.016^{*}$ \\
Weight $(\mathrm{g})$ & $497.7(457.0-538.5)$ & $447.0(392.5-501.4)$ & 22 & 0.135 \\
Specific Weight & $23.3(22.3-24.4)$ & $22.0(20.8-23.1)$ & 22 & 0.077 \\
\hline
\end{tabular}

BMI-Body mass index; * Significant at $\mathrm{p}<0.05$. 
Table 2: Coherent set of structural variables for determining oxygen diffusive conductance of the placenta

\begin{tabular}{|c|c|c|c|c|}
\hline Variables & Exposed & Non-exposed & df & p level \\
\hline \multicolumn{5}{|c|}{ Exchange surface area $\left(\mathrm{cm}^{2}\right)$} \\
\hline \multicolumn{5}{|l|}{ Maternal erythrocytes } \\
\hline \multicolumn{5}{|l|}{ Cotyledon region } \\
\hline Upper & $4.7 \mathrm{E} 5$ & $2.4 \mathrm{E} 5$ & 8 & $0.013 *$ \\
\hline Middle & $4.0 \mathrm{E} 5$ & $2.5 \mathrm{E} 5$ & 8 & 0.277 \\
\hline Lower & $3.2 \mathrm{E} 5$ & $3.7 \mathrm{E} 5$ & 8 & 0.781 \\
\hline \multicolumn{5}{|c|}{ Maternal throphoblast } \\
\hline Upper & $1.8 \mathrm{E} 5$ & $1.4 \mathrm{E} 5$ & 8 & 0.15 \\
\hline Middle & $1.6 \mathrm{E} 5$ & $2.8 \mathrm{E} 5$ & 8 & 0.398 \\
\hline Lower & $1.8 \mathrm{E} 5$ & $1.1 \mathrm{E} 5$ & 8 & $0.017 *$ \\
\hline \multicolumn{5}{|c|}{ Harmonic mean thickness } \\
\hline \multicolumn{5}{|c|}{ Foetal plasma } \\
\hline Upper & $1.5 \mathrm{E} 4$ & $9.6 \mathrm{E} 5$ & 8 & $0.012 *$ \\
\hline Middle & $1.1 \mathrm{E} 5$ & $9.2 \mathrm{E} 5$ & 8 & 0.286 \\
\hline Lower & $9.6 \mathrm{E} 5$ & $1.0 \mathrm{E} 4$ & 8 & 0.732 \\
\hline \multicolumn{5}{|c|}{ Partial diffusive conductance (ml/min/kPa) } \\
\hline \multicolumn{5}{|l|}{ Maternal plasma } \\
\hline Upper & 401.2 & 193.3 & 8 & $0.041 *$ \\
\hline \multicolumn{5}{|l|}{ Villous tissues } \\
\hline Lower & 352.8 & 197.3 & 8 & $0.027 *$ \\
\hline Foetal erythrocytes & 1149.4 & 815.3 & 8 & $0.049 *$ \\
\hline Lower & 1336.4 & 717.6 & 8 & $0.039 *$ \\
\hline \multicolumn{5}{|l|}{$\begin{array}{l}\text { Partial relative } \\
\text { resistances }(\%)\end{array}$} \\
\hline \multicolumn{5}{|l|}{ Foetal Plasma } \\
\hline Upper & 14.4 & 7 & 8 & $0.009 *$ \\
\hline Number of villi & 22 & 16 & 8 & $0.022 *$ \\
\hline Lower & 22.4 & 15.4 & 8 & $0.007 *$ \\
\hline
\end{tabular}

*Significant at $\mathrm{p}<0.05$. E: exponent to the power of $\left(10^{\mathrm{x}}\right)$ 